\title{
A Fuzzy Expert System for the Early Warning of Accidents Due to Driver Hypo- Vigilance
}

\author{
I. G. Damousis, D. Tzovaras and M. G. Strintzis \\ Informatics \& Telematics Institute \\ $1^{\text {st }} \mathrm{Km}$ Thermi-Panorama Road, PO Box 361, \\ GR-57001 Thermi-Thessaloniki, Greece \\ \{damousis, tzovaras\}@iti.gr
}

\begin{abstract}
In this paper a Fuzzy Expert System for the prediction of Hypovigilance-related accidents is presented. The system uses physiological modalities in order to detect signs of extreme hypovigilance. An advantage of such a system is its extensibility regarding the physiological modalities and features that it can use as inputs. In that way, even though currently only eyelid-related features are exploited, in the future and for prototypes designed for professionals other physiological modalities, such as EEG can be easily integrated in the existing system in order to make it more robust and reliable.
\end{abstract}

\section{Introduction}

The loss or the disruptions of sleep result in sleepiness during periods when the person should usually be fully awake. The loss of even one night's sleep can lead to extreme short-term sleepiness. The effects of sleep loss are cumulative and regularly losing one or two hours of sleep a night can result to chronic sleepiness over time

Sleep deprivation and related phenomena of excessive fatigue, prolonged inattention, hypovigilance and stress are among the key causes of serious industrial accidents such as nuclear accidents, chemical and environmental disasters, as well as fatal accidents [1].

An automated sleepiness monitoring system could watch over people to make sure the alertness and attention levels are high and warn or even take predefined measures when extreme hypovigilance is detected, in order to prevent an accident. This kind of system could increase the level of safety for everyone since it can be applied on a wide range or users, from regular drivers to sensitive equipment operators.

Several monitoring systems for the automatic hypovigilance detection have been developed over the past years [references]. The majority of those systems focus on

Please use the following format when citing this chapter:

Damousis, Ioannis, Tzovaras, Dimitrios, Strintzis, Michael, 2006, in IFIP International Federation for Information Processing, Volume 204, Artificial Intelligence Applications and Innovations, eds.

Maglogiannis, I., Karpouzis, K., Bramer, M., (Boston: Springer), pp. 345-352 
the diagnosis of the physiological demonstration of sleepiness, by recording and analyzing features that in most cases are related to the driver's blinking behavior.

Even though blink-related features intuitively and experimentally [2] seem to be the most suitable candidates for hypovigilance detection, studies show that these features are not enough accurate and reliable enough since they exhibit strong interpersonal (between persons) and intrapersonal (same person different times) variability. Aiming to address the limitations of the current hypovigilance detection and accident warning systems, we develop a new multimodal sleep prediction algorithm, which will be integrated in an automatic accident warning and sleep prediction prototype for drivers within the Integrated Project SENSATION.

The major objective of SENSATION is the development of new, unobtrusive sensors, capable of providing measurements that allow the online extraction of advanced physiological features that are not currently available in the existing warning systems. These features will potentially allow more accurate hypovigilance detection and the development of more reliable sleep prediction systems (less false warnings).

In this paper we describe the framework for such a multimodal physiological sleep prediction system, which is based on fuzzy logic expertise and trained with the use of real-coded Genetic algorithms. Also some preliminary results from the analysis of the training data, concerning the accident prediction effectiveness of blink-related features are reported.

\section{The Fuzzy Expert System in general (FES)}

Fuzzy logic is a research area based on the principles of approximate reasoning and computational intelligence. It departs from classical sets, logic and strict Boolean (True or False) decisions and assignments. Instead, it uses soft linguistic variables (e.g. small, medium, large), and a continuous range of truth-values in the interval $[0,1]$. Fuzzy models are employed in cases where a system is difficult to model exactly (but an inexact model is available), or ambiguity and vagueness is encountered in the problem formulation.

A typical fuzzy system comprises the following key parts:

- A rule base containing a number of IF-THEN rules,

- A fuzzy inference unit, which performs the inference operations of the rules

- The fuzzification interface which transforms crisp inputs into fuzzy variables that are processed by the fuzzy inference unit,

- The defuzzification interface that transforms the fuzzy output into a crisp number.

Expert knowledge can be "stored" in a fuzzy system's IF-THEN rules. This transfusion of knowledge in the system can take place either by the manual definition of the fuzzy rules, or by the training of the system using training cases or patterns. After the fuzzy rules are defined, the system is capable of making inferences and its output or decision simulates the one of an expert's. In that way the system is called Fuzzy Expert System.

Recently, the fuzzy inference system suggested by Takagi, Sugeno and Kang (TSK fuzzy model) has gained a great interest in several applications in fuzzy 
modelling and control. The TSK fuzzy models consist of linguistic fuzzy rules represented in the following form:

$$
\begin{aligned}
R^{(j)}: & \operatorname{IF}\left(x_{p, l} \text { is } A_{1}^{j}\right) A N D \ldots A N D\left(x_{p, N P I} \text { is } A_{N P I}^{j}\right) \\
& \operatorname{THEN} y_{j}=F_{j}\left(x_{c, l}, x_{c, 2}, \ldots, x_{c, N C l}\right) \quad j=1, \ldots, N R
\end{aligned}
$$

where $N R$ is the number of fuzzy rules.

The "IF" precondition statements define the premise part while the "THEN" rule functions constitute the consequent part of the fuzzy model.

$-\bar{X}_{p}=\left[x_{p, l}, \ldots, x_{p, N P I}\right]^{T}$ is the input vector to the premise part comprising $N P I$ input variables.

- $A_{i}$ are labels of fuzzy sets describing linguistically the input component $x_{p, i} i=$ $1, \ldots, N P I$. (e.g. "low", "medium", "high").

- $\bar{X}_{c}=\left[x_{c, l}, \ldots, x_{c, N C I}\right]^{T}$ denotes the input vector to the consequent part of $R^{(j)}$ containing $N C I$ input variables.

Finally, $y_{j}=F\left(\bar{X}_{c}\right)$ represents the $j$-th rule output which is a function of the consequence part input components $x_{c, i}, i=1, \ldots, N C l$. A special case of particular importance is encountered when the rule functions are linear polynomials of the consequent inputs:

$$
y_{j}=F\left(\bar{X}_{e}\right)=\lambda_{0}^{j}+\sum_{i=1}^{N C I} \lambda_{i}^{j} x_{c, i}
$$

where $\lambda_{i}^{j}$ are weight coefficients and $\lambda_{0}^{j}$ is a bias term.

Each linguistic label $A_{i}^{j}$ is associated with a membership function $\mu_{i}^{j}\left(x_{p, i}\right)$. These are usually unimodal functions (triangular, Gaussian, bell shaped, etc.), taking values in the interval $[0,1]$. Gaussian type memberships are employed described by

$$
\mu_{i}^{j}\left(x_{p, i}\right)=\exp \left[-\frac{1}{2} \frac{\left(x_{p, i}-m_{i}^{j}\right)^{2}}{\left(\sigma_{i}^{j}\right)^{2}}\right]
$$

where $m_{i}^{j}$ and $\sigma_{i}^{j}$ are the mean value and the standard deviation of the membership function, respectively (Fig. 1 (a)).

The firing strength of the rule $R^{(j)}$, representing the degree to which $R^{(j)}$ is excited by a particular premise input vector $\bar{X}_{p}$, is determined by

$$
\mu_{j}\left(\bar{X}_{c}\right)=\prod_{i=1}^{N P I} \mu_{i}^{j}\left(x_{p, i}\right) .
$$

The antecedent fuzzy sets pertaining to a rule $R^{(j)}$ define a fuzzy region within the premise space (Fig. 1 (b))

$$
\mathbf{A}^{(j)}=A_{1}^{j} \times A_{2}^{j} \times \cdots \times A_{N P I}^{j}
$$

Essentially, $\mathbf{A}^{(j)}$ represents a multidimensional fuzzy set with a membership distribution defined by (4). 
Using this notation, the TSK rule can be brought in the following compact form:

$$
R^{(j)}: \operatorname{IF} \bar{X}_{p} \text { is } A^{(j)} \operatorname{THEN} y_{j}=F_{j}\left(\bar{X}_{c}\right)
$$

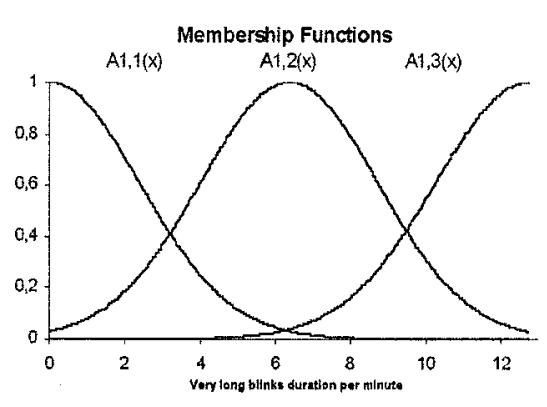

(a)

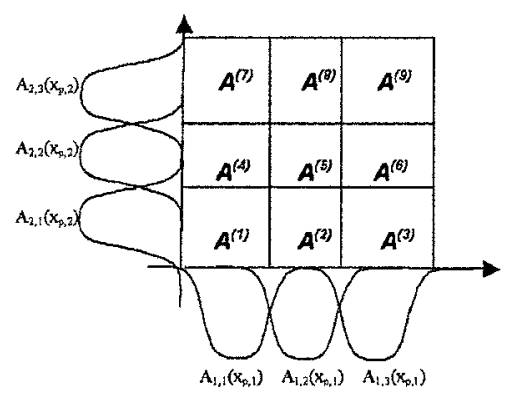

(b)

Fig.1. (a) Assuming the "very long blinks duration per minute" feature is a premise input, $x_{p, l}$, three fuzzy sets $A_{l, 1}, A_{l, 2}$ and $A_{l, 3}$ can express the linguistic propositions that the measured "very long blinks duration per minute" is "Low", "Medium" or "High", respectively. Thus, for a specific sample $x_{p, l}=4 \mathrm{sec}$ the memberships for each of the fuzzy sets are $0.2,0.62,0.0$ respectively and the measured "very long blinks duration" is linguistically described as "medium to low". (b) Three membership functions $A_{i, 1}\left(x_{p, i}\right), A_{i, 2}\left(x_{p, i}\right)$, and $A_{i, 3}\left(x_{p, i}\right)$ are used for each premise input $i$, to express linguistic properties of the inputs, forming nine fuzzy regions that define the boundaries of the system's fuzzy rules.

Given the input vectors $\bar{X}_{p}$ and $\bar{X}_{c}$, the final output of the fuzzy model is inferred using the weighted average defuzzification method [12] as follows

$$
y=\frac{\sum_{j=1}^{N R} \mu_{j}\left(\bar{X}_{p}\right) \cdot F_{j}\left(\bar{X}_{c}\right)}{\sum_{j=l}^{N R} \mu_{j}\left(\bar{X}_{p}\right)}
$$

From the above description, it can be seen that the basic philosophy of the TSK model is to decompose the premise space into fuzzy regions $\mathrm{A}^{(j)}$ and approximate the system's behaviour in every region by a simple submodel $F\left(\bar{X}_{c}\right)$. Thus, the overall model can be regarded as a fuzzy blending of linear submodels with simpler structure.

\section{Accident Prediction Fuzzy Expert System}

Our objective is to develop a TSK fuzzy model that provides early warnings for accidents that are due to driver's hypovigilance or sleep onset, based on 
physiological features. The fuzzy decomposition of the premise space should allow the discrimination between different physiological demonstrations of extreme sleepiness and address the inter-personal variability. In order though for such a system to be efficient and to model (in order to detect) all the different ways that people exhibit extreme hypovigilance just before the sleep onset, we have to select the appropriate physiological features that describe adequately these ways.

\subsection{Selection of the physiological inputs}

To construct the fuzzy model structure, a number of premise inputs $x_{p,}, \ldots, x_{p, N P I}$ should be properly selected. These are the decision variables that constitute the premise space and will allow the formulation of rules (discrete cases). Each premise variable will then partitioned by a certain number of fuzzy sets that cover adequately its universe of discourse as shown in Fig.1(a).

The number of premise inputs should be as small as possible. A reasonable choice is to select one or two inputs. This is dictated by our requirement to keep the number of rules to an acceptably low level. However the great inter-personal variability of the physiological signs that characterize the phase prior to sleep onset may require the use of several features that will serve as FES premise inputs in order to define as accurately as possible all the different classes of physiological behaviours prior to falling asleep. There are several studies in the literature that aim to determine the appropriate physiological signals that allow hypovigilance diagnosis from a broad set of candidate inputs [5], [6], however most of them are inconclusive and there seems to be no golden standard in feature selection or combination of features that can lead to a full proof prediction system.

The physiological features that are related to hypovigilance are EEG features such as alpha and theta waves, eyelid activity features such as long blinks, eye activity related features such as slow eye movements (SEM) and pupillography.

However, since EEG and SEM data can only be acquired via electrodes, they cannot be used for online predictions due to restrictions stemming from user unobtrusiveness requirements. Because of this, EEG analysis is only used as a reference and we can only utilize eyelid activity features (blinks) that can be recorded unobtrusively with CMOS cameras.

For the proposed FES, the decision on the blink-related features selection was taken following a two-steps process:

1) Literature review study in order to pinpoint the most promising features for the discrimination of the various behaviours prior to sleep [5],[6] and also following the guidelines over the use of various physiological for hypovigilance diagnosis and sleep prediction provided by [7],[9].

2) Experimental parametric analysis of the above features using real driving data from 37 subjects [3], in order to select the features with the highest correlation to accidents (Fig.2). 

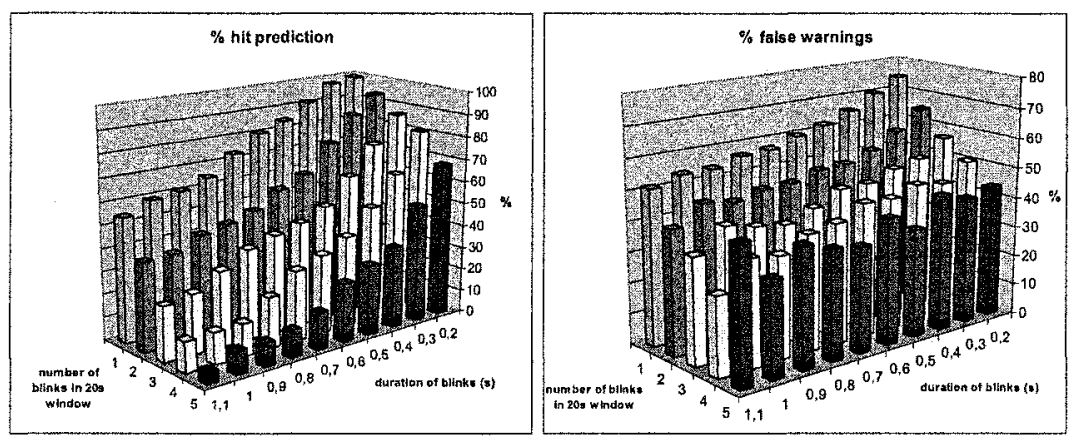

Fig. 2. Parametric analysis of the "number of long blinks" feature. The variables are the duration of a "long blink" and the number of long blinks during a 20 second window that slides every five seconds. Even though the sensitivity of the feature is good (e.g. when 5 detected blinks with duration over $0,2 \mathrm{~s}$ around $70 \%$ of the hits are predicted within the next 2 minutes), the specificity of the system is not acceptable (more than $40 \%$ of the warnings are inaccurate).

\section{Genetic Algorithm (GA) training of the FES parameters}

The objective of the FES training is to set the values of the premise and consequence part variables in such way as to predict as accurately as possible the accidents, based on the eyelid-related features that are used as inputs. The training patterns have the following structure:

$$
\left\lfloor\bar{X}_{p}\left|\bar{X}_{c}\right| Y_{A C C I D E N T}\right\rfloor
$$

where $\bar{X}_{p}$ and $\bar{X}_{c}$ are the input vectors to the premise and the consequent part respectively (blink-related features) and $Y_{A C C I D E N T}$ is a binary value that indicates whether an accident happened at that moment ("1") or not ("0"). We must note here that the accidents are filtered based on EEG and EOG analysis in order to take into considerations only those accidents that are due to hypovigilance [4].

For the training of the accident prediction FES a real-coded GA is used. For this GA implementation the parameters of the premise and the consequence parts are concatenated in order to form a genotype or chromosome which is a consolidated representation of a FES. The premise parameters are the mean values and standard deviations of the membership functions that partition the premise inputs. These variables define fully the membership functions and also set the boundaries of the fuzzy rules (the $I F$ part of the rules).

The consequence part parameters are the $\lambda_{i}^{j}, i=0, \ldots, N C I, j=1, \ldots, N R$ coefficients that define the output of each fuzzy rule as shown in (7).

All training parameters, as well as the training patterns' data are normalized in the $[0,1]$ space. An obvious advantage of the real-coded GA over binary-coded GAs is 
that with the direct encoding of floating-point numbers in the chromosomes we achieve absolute precision, overcoming the critical decision of the number of bits to be used for the encoding of8each FES parameter.

The training process of the FES using GA begins with the random generation of an initial population of $m$ genotypes. The quality of the solution that a specific genotype represents is measured by calculating its fitness following the next steps:

a) Decomposition of the chromosome into FES premise and consequence parameters

b) Calculation of FES output for each training pattern

Equation (3) provides the memberships of the training pattern to the fuzzy sets that partition the premise inputs. Then the pattern's firing strength for each fuzzy rule is calculated (4). Each rule has an output that corresponds to the specific pattern as is shown in (2). The overall output of the FES for the specific pattern is the weighted average of the fuzzy rules' outputs as shown in (7). Each rule's contribution to the final solution is analogous to the degree that the pattern triggers the specific rule.

c) Calculation of the chromosome's fitness

The FES output is compared with a threshold. The threshold is also part of the chromosome, hence trainable as well. If the output of the FES is larger than the threshold then the expert system produces an accident warning ("1"). If not, the system's output is " 0 ". The outputs of the system are compared to the actual accidents and a measure of accuracy is calculated:

$$
\text { FitnessFunction }=\frac{1+\operatorname{shp}(\%)}{1+\operatorname{far}(\%)}
$$

Where $\operatorname{sh} p(\%)$ is the successful hit prediction ratio, defined as the percentage of hits that were predicted and $\operatorname{far}(\%)$ is the false alarms ratio which is defined as the percentage of FES warnings that did not correspond to an accident up to 2 minutes ahead. As it can be seen from (8) this fitness function promotes the sensitivity (promoting accurate predictions) and the specificity of the system (false alarms).

The GA is allowed to evolve for a number of generations. The evolution takes place using the well-known genetic operators of selection, crossover [9] and mutation [8]. The final FES derives from the elite solution of the GA at the final generation. Upon termination of the training process, the quality of the obtained model is verified with the testing data set. While GA training lasts from minutes to some hours, depending on the size of the measurements database, the on-line predictions that are based on real time measurements are attained instantly.

\section{Experimental results and Conclusions}

A FES was developed as described in Sections 2, 3 and 4. However because the feature extraction process is currently not concluded, only blink-related features that are available by conventional low frame rate cameras were used. As it was mentioned in section 3.1 , the only blink-related feature that exhibited adequate 
sensitivity and specificity was the number of long-blinks feature. In order to present this feature as a fuzzy premise input, we used the duration of the "long blinks" that were detected during the previous 20 second window. Based on the findings of section 3.1 we defined the duration that characterizes a long blink, using the sensitivity/specificity ratio as criterion. In that special case that we only use one feature to create our system, the FES can only be considered as a fine-tuning method based on AI techniques, aiming to maximize sensitivity (prediction accuracy) and specificity (false alarms minimization). This process led to $72 \%$ accuracy in hit prediction accompanied by $32 \%$ false alarms. Future work includes the study and integration of new more advanced eyelid-activity related features when they are available, in order to develop a more reliable accident prediction system. These features include PERCLOS [2], amplitude and peak closing velocity as well as lid closure and opening speed [6]. Eye gaze features are also being extracted in order to provide information about fixations that usually accompany extreme hypovigilance. A second step will be the integration of EEG features such as alpha and theta waves for the development of a sleep prediction system for professionals, where sensor unobtrusiveness is less important than reliability.

These new features can be easily integrated due to the open structure of the FES and the flexibility of the GA training as opposed to the various mathematical models.

\section{References}

[1] Akerstedt et al., "Work organisation and unintentional sleep: results from the WOLF study" Occup Environ Med 2002;59:595-600.

[2] Dinges, Mallis, et al.: Evaluation of techniques for ocular measurement as an index of fatigue and the basis for alertness management. Final report for the USDOT, NHTSA, 104pp, Report No. DOT HS 808 762, 1998

[3] Björn Peters, Anna Anund, Joakim Östlund and Magnus Hjälmdahl, Results of Sensation Pilot 2.5 - WP1.7 (Alertness Monitoring Database), SENSATION internal deliverable, Nov. 2005.

[4] Rechtschaffen, A., \& Kales, A. (1968). A manual of standardized terminology, techniques and scoring system for sleep stages of human subjects. Bethesda: US Department of Health, Education and Welfare, Public Health Service.

[5] N.Galley, R.Schleicher, L.Galley, "Blink parameter for sleepiness detection" and other works by the same authors.

[6] Caffier, P.P., Erdmann, U., Ullsperger, P. Experimental evaluation of eye-blink parameters as a drowsiness measure. Eur J Appl Physiol, 89: 319-325. 2003.

[7] Yannis Damousis, Dimitrios Tzovaras: Correlation between SP1 data and parameters and WP 4.4.2 algorithms, Sensation Internal Report (Draft Nov2004)

[8] Z. Michalewicz, Genetic Algorithms + Data Structures = Evolution Programs, New York / USA, Springer-Verlag, 1996.

[9] F. Herrera, M. Lozano, J.L. Verdegay, Tuning fuzzy controllers by genetic algorithms, Internat. J. Approx. Reasoning 12 (1995) 299-315.

[10] Alex H. Bullinger et al "Criteria and algorithms for physiological states and their transitions, SENSATION_Del_1_1_1.doc", SENSATION Deliverable 1.1.1, August 2004. 